\title{
On Second-Order Necessary Conditions in Optimal Control of Problems with Mixed Final Point Constraints
}

\author{
Helene Frankowska ${ }^{1}$
}

\begin{abstract}
We investigate the second-order necessary optimality conditions for weak local minima for the Mayer optimal control problem with an arbitrary control constraint $U \subset \mathbb{R}^{m}$ and final-point constraints given by equalities and inequalities. In the difference with the previous literature, we do not impose structural assumptions on $U$ and use an inverse mapping theorem on a metric space to derive a variational inequality. The separation theorem leads in a straightforward way to the second-order necessary optimality conditions.
\end{abstract}

\section{INTRODUCTION}

We address here the Mayer optimal control problem

$$
\begin{aligned}
& \text { Minimize } \varphi(x(1)) \\
& g_{i}(x(1)) \leq 0, \quad i=1, \ldots, r \\
& h_{j}(x(1))=0, \quad j=1, \ldots, k \\
& \dot{x}(t)=f(x(t), u(t)), \quad x(0)=x_{0}, \quad u(t) \in U \text { a.e. }
\end{aligned}
$$

where $U$ is an arbitrary nonempty compact subset of $\mathbb{R}^{m}$, $x_{0} \in \mathbb{R}^{n}$ is given and functions $g_{i}: \mathbb{R}^{n} \rightarrow \mathbb{R}, h_{j}: \mathbb{R}^{n} \rightarrow \mathbb{R}$, $f: \mathbb{R}^{n} \times \mathbb{R}^{m} \rightarrow \mathbb{R}^{n}$ are twice continuously differentiable.

It is well known that the Bolza problem involving also an integral functional in the cost can be reduced to the above Mayer problem by adding an extra state variable.

Our aim is to derive the second-order necessary optimality conditions for merely measurable optimal controls. The literature on second-order necessary optimality conditions often involves structural assumptions on optimal controls, as for instance their piecewise continuity and, usually, the set $U$ is described by relations verifying some constraint qualifications involving the space $L^{\infty}\left([0,1], \mathbb{R}^{m}\right)$, see for instance [3], [11], [14]. We would like to recall that the existence theorems in optimal control theory, in general, guarantee only measurability of optimal controls. To get their piecewise continuity very strong coercivity assumptions have to be added. On the other hand, the literature on the first-order necessary conditions usually deals with merely measurable optimal controls and concerns very general situations when the control set $U$ is arbitrary. So an important gap was created between the achievements in the first-order and the secondorder conditions.

For this reason new variational tools were developed recently in [10], [5], [6], [7] to address the general case. These tools rely on the first and second-order linearizations

\footnotetext{
*This material is based upon work supported by the Air Force Office of Scientific Research under award number FA9550-18-1-0254.

1 Helene Frankowska is with CNRS, IMJ-PRG, Sorbonne Université, case 247, 4 place Jussieu, 75252 Paris, France helene.frankowska@imj-prg.fr
}

of control systems and the separation theorems. The method is so straightforward that it allowed also to study first and second-order optimality conditions for the stochastic optimal control problems [8], [9]. Nevertheless, an interesting case of the final state constraints involving equalities was left aside because it was not clear yet what tools to apply to deal with it. The aim of this paper is to show how this case can be investigated using an inverse mapping theorem on a metric space.

The results below can be derived as well when initial point constraints, non-autonomous control systems and nonautonomous control constraint $u(t) \in U(t)$ are involved. Here we avoid such more general setting for the sake of simplicity.

In this paper only weak local minimizers in $L^{1}([0,1], U)$ are investigated. Note that under our assumptions below any strong local minimizer, see for instance [7] for the definition, is also a weak local minimizer. Some authors addressed second-order necessary optimality conditions for weak local minimizers in $L^{\infty}([0,1], U)$, when the set $U$ and the optimal control have a particular structure, cf. [1], [3]. However this space offers weaker results than $L^{1}([0,1], U)$, because, for instance, classical perturbations by needle variations are large in $L^{\infty}([0,1], U)$, while being small in $L^{1}([0,1], U)$.

We provide here a second-order necessary optimality condition in the same spirit as in [7] under an additional end point equality constraints. Our main result, Theorem 1 below, involves a (finite dimensional) constraint qualification on the reachable set of the linear system (11). When it is not satisfied, then an abnormal maximum principle holds true. If the control set $U$ has a particular structure described by inequalities with linearly independent gradients of active constraints such qualification assumption is not needed, cf. [13]. It is an interesting open question if this qualification assumption can be skipped in a general situation as well.

Traditionally, in the presence of the end-point equality constraints, various authors use the classical implicit function theorem on the space of controls. The main difficulty that one faces when $U$ does not have a particular structure is the lack of such a result. Below we provide a new implicit function theorem, Theorem 4, on the metric space $L^{1}([0,1], U)$, which is well adapted to deal with the end-point equality constraints.

The paper is organized as follows. In Section II we introduce some notations and recall the notions of first and second-order tangents. The main results are stated in Section III, while their proofs are given in Section V. Section IV is devoted to an implicit function theorem that allows to prove 
a variational equation under end-point equality constraints.

Because of the lack of space, we do not overview here the earlier literature concerning the second-order optimality conditions. The interested reader will find in [14] further references and the historical comments.

\section{PRELIMINARIES}

We denote by $\mathbb{R}_{+}$the set of all reals $\gamma \geq 0$. In this paper $|\cdot|$ and $\langle\cdot, \cdot\rangle$ stand for the Euclidean norm and the scalar product in a finite dimensional space. Denote by $W^{1,1}\left([0,1], \mathbb{R}^{n}\right)$ the Sobolev space of absolutely continuous functions $x$ : $[0,1] \rightarrow \mathbb{R}^{n}$, by $\|\cdot\|_{\infty}$ the supremum norm on the space of continuous functions $C\left([0,1], \mathbb{R}^{n}\right)$, by $L^{1}\left([0,1], \mathbb{R}^{m}\right)$ the space of Lebesgue integrable functions $u:[0,1] \rightarrow \mathbb{R}^{m}$ with the norm $\|u(\cdot)\|_{1}=\int_{0}^{1}|u(t)| d t$ and by $L^{\infty}\left([0,1], \mathbb{R}^{m}\right)$ the space of measurable, essentially bounded functions $u$ : $[0,1] \rightarrow \mathbb{R}^{m}$. The following notation will be used:

$$
\Xi:=W^{1,1}\left([0,1], \mathbb{R}^{n}\right) \times L^{\infty}\left([0,1], \mathbb{R}^{m}\right) .
$$

Any trajectory-control pair $(x, u) \in \Xi$ satisfying (2)-(4) is called admissible. An admissible $(\bar{x}, \bar{u})$ is a weak local minimizer (in $L^{1}$ ) if for some $\varepsilon>0$ we have $\varphi(x(1)) \geq \varphi(\bar{x}(1)$ ) for any admissible $(x, u) \in \Xi$ such that $\|u-\bar{u}\|_{1}<\varepsilon$.

The Hamiltonian and the terminal Lagrange function are defined respectively by $\mathcal{H}(x, u, p)=\langle p, f(x, u)\rangle$ and

$$
l(x, \alpha, \beta)=\alpha_{0} \varphi(x)+\sum_{i=1}^{r} \alpha_{i} g_{i}(x)+\sum_{j=1}^{k} \beta_{j} h_{j}(x),
$$

where $\alpha=\left(\alpha_{0}, \alpha_{1}, \ldots, \alpha_{r}\right) \in \mathbb{R}^{r+1}, \beta=\left(\beta_{1}, \ldots, \beta_{k}\right) \in \mathbb{R}^{k}$. Set $h(x):=\left(h_{1}(x), \ldots, h_{k}(x)\right), K_{i}:=\left\{x \in \mathbb{R}^{n}: g_{i}(x) \leq\right.$ $0\}$ for $i=1, \ldots, r$ and observe that for every $x \in \partial K_{i}$ we have $g_{i}(x)=0$.

Let $X$ be a Banach space and $\emptyset \neq K \subset X$. We denote by Int $K, \partial K, \overline{c o} K$, respectively, its interior, its boundary and its closed convex hull. The distance from $x \in X$ to $K$ is defined by $\mathrm{d}_{K}(x)=\inf _{x^{\prime} \in K}\left|x^{\prime}-x\right|$. The adjacent tangent cone to $K$ at $\bar{x} \in K$ is the closed cone

$$
T_{K}^{b}(\bar{x})=\left\{v \in X \mid \mathrm{d}_{K}(\bar{x}+\delta v)=o(\delta), \forall \delta>0\right\}
$$

and the second-order adjacent set to $K$ at $(\bar{x}, v) \in K \times X$ is the closed set

$T_{K}^{b(2)}(\bar{x}, v)=\left\{w \in X \mid \mathrm{d}_{K}\left(\bar{x}+\delta v+\delta^{2} w\right)=o\left(\delta^{2}\right), \forall \delta>0\right\}$,

where the vectors $o(s) \in X$ are so that $\lim _{s \rightarrow 0+} \frac{1}{s} o(s)=0$. See [2] for some properties and calculus of the first and second-order tangent sets.

The Clarke tangent cone to $K$ at $x \in K$ is the set of all $v \in X$ such that for any sequence $x_{i} \in K$ converging to $x$ and reals $h_{i} \rightarrow 0+$, there exist $v_{i} \in X$ converging to $v$ such that $x_{i}+h_{i} v_{i} \in K$. It was shown in [5] that

$$
T_{K}^{b(2)}(\bar{x}, v)+C_{K}(\bar{x})=T_{K}^{b(2)}(\bar{x}, v) .
$$

For a family $\left\{A_{\delta}\right\}_{\delta>0}$ of subsets of $X$ the PeanoKuratowski upper set limit is defined by

$$
v \in \operatorname{Limsup}_{\delta \rightarrow 0+} A_{\delta} \Leftrightarrow \liminf _{\delta \rightarrow 0+} \mathrm{d}_{A_{\delta}}(v)=0 .
$$

\section{MAIN RESULTS}

The following assumptions will be used below.

$$
\text { (H1) } \exists \gamma>0, \sup _{u \in U}|f(x, u)| \leq \gamma(|x|+1) \forall x \in \mathbb{R}^{n},
$$

$(H 2)\left\{\begin{array}{l}f \text { is continuous, differentiable with respect to } x \\ \text { and } f_{x}(\cdot, \cdot) \text { is continuous. }\end{array}\right.$

Let $(\bar{x}, \bar{u})$ be a weak local minimizer for problem (1)-(4). Define the set of active indices $I_{g}:=\left\{i: \bar{x}(1) \in \partial K_{i}\right\}$.

When $\varphi, f, h, g_{i} \in C^{1}$ for $i=1, \ldots, r$, the first-order necessary condition for weak local minimizers is as follows: there exist $\beta=\left(\beta_{1}, \ldots, \beta_{k}\right) \in \mathbb{R}^{k}$ and $\alpha=\left(\alpha_{0}, \ldots, \alpha_{r}\right) \in$ $\mathbb{R}_{+}^{r+1}$, not vanishing simultaneously, satisfying

$$
\alpha_{i}=0 \text { if } i \notin I_{g}
$$

such that for the solution $p \in W^{1,1}\left([0,1], \mathbb{R}^{n}\right)$ of

$$
\begin{array}{r}
-\dot{p}(t)=\mathcal{H}_{x}(\bar{x}(t), \bar{u}(t), p(t)) \quad \text { a.e. } \\
p(1)=l_{x}(\bar{x}(1), \alpha, \beta)
\end{array}
$$

we have

$$
\inf _{\kappa \in C_{U}(\bar{u}(t))} \mathcal{H}_{u}(\bar{x}(t), \bar{u}(t), p(t)) \kappa \geq 0 \quad \text { a.e. }
$$

where $\mathcal{H}_{x}$ and $\mathcal{H}_{u}$ stand for the derivatives of $\mathcal{H}$ with respect to $x$ and $u$. This necessary condition can be proved using the separation theorem, similarly to the proof of the secondorder condition provided below, under less regularity of data. Since in this paper we are interested by the second-order conditions, we shall derive the first- order condition as a consequence of the second-order one and, so, for $C^{2}$ data.

Denote by $\Lambda(\bar{x}, \bar{u})$ the set of all $0 \neq(\alpha, \beta, p) \in$ $\mathbb{R}_{+}^{r+1} \times \mathbb{R}^{k} \times W^{1,1}\left([0,1], \mathbb{R}^{n}\right)$ satisfying (6)-(9) and set $[t]:=(\bar{x}(t), \bar{u}(t))$.

For a continuously differentiable $f$ consider the classical variational linearization of control system $(4)$ at $(\bar{x}, \bar{u})$ :

$$
\left\{\begin{array}{l}
\dot{y}(t)=f_{x}[t] y(t)+f_{u}[t] u(t), u(t) \in T_{U}^{b}(\bar{u}(t)) \\
y(0)=0,
\end{array}\right.
$$

where $f_{x}, f_{u}$ denote the derivatives of $f$ with respect to $x$ and $u$ and $(y, u) \in \Xi$.

The second type of linearization of (4) at $(\bar{x}, \bar{u})$ is as follows:

$$
\left\{\begin{array}{l}
\dot{y}(t)=f_{x}[t] y(t)+v(t), v(t) \in f(\bar{x}(t), U)-f[t] \\
y(0)=0,
\end{array}\right.
$$

where $(y, v) \in \Xi$. The reachable set of (11) at time 1 is

$$
R^{L}(1)=\{y(1): y(\cdot) \text { is a trajectory of }(11)\} .
$$

It is well known that, under assumptions (H1), (H2) the set $R^{L}(1)$ is convex and compact.

Let $\mathcal{C}(\bar{x}, \bar{u})$ be the set of all pairs $(y, u) \in \Xi$ solving the linear system (10) such that $\varphi^{\prime}(\bar{x}(1)) y(1) \leq 0$,

$$
\begin{aligned}
& h^{\prime}(\bar{x}(1)) y(1)=0, g_{i}^{\prime}(\bar{x}(1)) y(1) \leq 0, \quad \forall i \in I_{g}, \\
& \left\{\begin{array}{l}
\exists \delta_{0}>0, \quad \exists c \geq 0 \quad \text { such that } \forall \delta \in\left[0, \delta_{0}\right] \\
\mathrm{d}_{U}(\bar{u}(t)+\delta u(t)) \leq c \delta^{2} \quad \text { for a.e. } t \in[0,1] .
\end{array}\right.
\end{aligned}
$$


$\mathcal{C}(\bar{x}, \bar{u})$ is called in this paper the critical cone.

The proof of the next lemma follows by arguments similar to [6, Proof of Lemma 3.2] and is omitted.

Lemma 1: Let $\varphi, f, h, g_{i} \in C^{1}$ for $i=1, \ldots, r$ and $(\alpha, \beta, p) \in \Lambda(\bar{x}, \bar{u})$. Then for any $(y, u) \in \mathcal{C}(\bar{x}, \bar{u})$, we have $\alpha_{i} g_{i}^{\prime}(\bar{x}(1)) y(1)=0, i=1, \ldots, r \quad \& \quad \mathcal{H}_{u}[t] u(t)=0 \quad$ a.e., where $\mathcal{H}_{u}[t]$ denotes the gradient of $\mathcal{H}(\bar{x}(t), \cdot, p(t))$ at $\bar{u}(t)$.

Assume next that $f$ is twice continuously differentiable. To express the second-order optimality conditions, we use the second-order tangents. We associate with any $u:[0,1] \rightarrow$ $\mathbb{R}^{m}$ the set

$$
\begin{aligned}
& V^{2}(\bar{u}, u):=\left\{v:[0,1] \rightarrow \mathbb{R}^{m}: v(\cdot)\right. \text { is measurable, } \\
& \left.f_{u}[\cdot] v(\cdot) \text { is integrable and } v(t) \in T_{U}^{b(2)}(\bar{u}(t), u(t)) \text { a.e. }\right\} .
\end{aligned}
$$

For every $(\alpha, \beta, p) \in \Lambda(\bar{x}, \bar{u})$ and $t \in[0,1]$, define

$$
\Upsilon(u(t), p(t)):=\inf \left\{\mathcal{H}_{u}[t] v: v \in T_{U}^{b(2)}(\bar{u}(t), u(t))\right\},
$$

where, by convention, $\inf _{\emptyset}=+\infty$.

For any $(\alpha, \beta, p) \in \Lambda(\bar{x}, \bar{u})$ and $\xi=(y, u) \in \Xi$, define

$$
\begin{aligned}
\Omega(\xi, \alpha, \beta, p):= & y(1)^{*} l_{x x}(\bar{x}(1), \alpha, \beta) y(1)+ \\
& \int_{0}^{1} \xi(t)^{*} \mathcal{H}^{\prime \prime}[t] \xi(t) \mathrm{d} t,
\end{aligned}
$$

where $\mathcal{H}^{\prime \prime}[t]$ is the Hessian of $\mathcal{H}(\cdot, \cdot, p(t))$ at $(\bar{x}(t), \bar{u}(t))$.

Our main result is the following theorem.

Theorem 1: Assume (H1) and that $f, \varphi, h, g_{i} \in C^{2}$ for $i=1, \ldots, r$. Let $(\bar{x}, \bar{u})$ be a weak local minimizer satisfying

$$
0 \in \operatorname{Int} h^{\prime}(\bar{x}(1))\left(R^{L}(1)\right)
$$

and $\xi=(y, u) \in \mathcal{C}(\bar{x}, \bar{u})$ be such that the set $V^{2}(\bar{u}, u)$ contains an essentially bounded function. Then for some $(\alpha, \beta, p) \in \Lambda(\bar{x}, \bar{u})$, the function $\Upsilon(u, p)$ is integrable and

$$
\frac{1}{2} \Omega(\xi, \alpha, \beta, p)+\int_{0}^{1} \Upsilon(u(t), p(t)) \mathrm{d} t \geq 0 .
$$

Remark 1: (a) In the absence of equality constraints, the proof of Theorem 1 provided below still implies the above result without any constraint qualification like (12). However such case was already investigated in [7].

(b) The interested reader can find in [6], [7] several examples, where, for every critical element $(x, u) \in \mathcal{C}(\bar{x}, \bar{u})$, the condition $V^{2}(\bar{u}, u) \cap L^{\infty}\left([0,1], \mathbb{R}^{m}\right) \neq \emptyset$ is fulfilled.

(c) Observe that if $h^{\prime}(\bar{x}(1))$ is not surjective, then there exists $0 \neq q \perp \operatorname{Im} h^{\prime}(\bar{x}(1))$. Thus $h^{\prime}(\bar{x}(1))^{*} q=0$. Let $\xi=(y, u) \in \mathcal{C}(\bar{x}, \bar{u})$. If $\left\langle l_{x x}(\bar{x}(1), 0, q) y(1), y(1)\right\rangle \geq 0$, then set $\beta=q$. If $\left\langle l_{x x}(\bar{x}(1), 0, q) y(1), y(1)\right\rangle<0$, then define $\beta=-q$. For such $\beta$ and $\alpha=0$, we have $p \equiv 0$. Thus $\mathcal{H}_{u}[t]=0$ and relations (6)-(9) are satisfied. So the secondorder necessary condition is $\Omega(\xi, 0, \beta, p) \geq 0$. Though it is in line with the necessary condition of Theorem 1 , it does not contain any relevant information about the optimal control. Thus only the case of surjective $h^{\prime}(\bar{x}(1))$ is of interest.

(d) Relation (12) can be equivalently stated by saying that the image by $h^{\prime}(\bar{x}(1))$ of the convex cone spanned by $R^{L}(1)$ is the whole space. Denote by $\mathcal{A}^{L}(1)$ the reachable set of the classical linearized system (10). Then this set is a cone. By not difficult, but lengthly calculations, it is possible to verify that if $h^{\prime}(\bar{x}(1))\left(\mathcal{A}^{L}(1)\right)=\mathbb{R}^{n}$, then (12) is satisfied. The converse is not, in general, true because (11) involves the whole set $f(\bar{x}(t), U)$ and not just its local approximations via tangents. For this reason $R^{L}(1)$ allows to state a weaker assumption than $\mathcal{A}^{L}(1)$.

(e) As we show in the proof of Theorem 3 below, the failure of (12) leads to an abnormal maximum principle not involving the cost function, even, more precisely, with $\alpha=0$. Consequently, in this case, even the first- order optimality condition becomes a relation between the reachable set $R^{L}(1)$ and the image of $h^{\prime}(\bar{x}(1))$ rather than an optimality statement.

Recall that if $\alpha_{0}>0$, then the multiplier rule in Theorem 1 is called normal and, by normalizing, one can put $\alpha_{0}=$ 1. Normality of the necessary conditions is of a crucial importance, because otherwise they do not depend on the cost function. We propose next a second-order sufficient condition for normality.

Denote by $f^{\prime \prime}$ the Hessian of $f$. Fix a trajectory control pair $\xi=(y, u) \in \Xi$ of (10). We associate with it a secondorder linearization

$$
\left\{\begin{array}{l}
\dot{w}(t)=f_{x}[t] w(t)+f_{u}[t] v(t)+\frac{1}{2} \xi(t)^{*} f^{\prime \prime}[t] \xi(t) \\
v(t) \in T_{U}^{b(2)}(\bar{u}(t), u(t)) \text { a.e. } \\
w(0)=0
\end{array}\right.
$$

where $(w, v) \in \Xi$. Denote by $R^{L(2)}(1)$ the reachable set at time 1 of (14). By the Aumann theorem it is convex.

We also consider the linear control system

$$
\left\{\begin{array}{l}
\dot{z}(t)=f_{x}[t] z(t)+f_{u}[t] \pi(t), \pi(t) \in C_{U}(\bar{u}(t)) \\
z(0)=0,
\end{array}\right.
$$

where $(z, \pi) \in \Xi$ and denote by $\mathcal{R}(1)$ the reachable set of (15) at time 1 . Clearly it is a convex cone containing 0.

Theorem 2: Assume (H1) and that $f, \varphi, h, g_{i} \in C^{2}$ for $i=1, \ldots, r$. Let $(\bar{x}, \bar{u})$ be a weak local minimizer satisfying

$$
h^{\prime}(\bar{x}(1))(\mathcal{R}(1))=\mathbb{R}^{n}
$$

and $(y, u) \in \mathcal{C}(\bar{x}, \bar{u})$ be so that $V^{2}(\bar{u}, u) \cap L^{\infty}\left([0,1], \mathbb{R}^{m}\right) \neq$ $\emptyset$. If there exists $w(1) \in R^{L(2)}(1)$ such that for every $j \in$ $\{1, \ldots, k\}$,

$$
h_{j}^{\prime}(\bar{x}(1)) w(1)+\frac{1}{2} y(1)^{*} h_{j}^{\prime \prime}(\bar{x}(1)) y(1)=0
$$

and

$$
g_{i}^{\prime}(\bar{x}(1)) w(1)+\frac{1}{2} y(1)^{*} g_{i}^{\prime \prime}(\bar{x}(1)) y(1)<0 \quad \forall i \in I_{g},
$$

then the conclusion of Theorem 1 is valid with $\alpha_{0}=1$.

We next observe that if there is no $w(1) \in R^{L(2)}(1)$ as in the assumption of the above theorem, then the second-order condition holds true in the abnormal form.

Proposition 1: Assume (H1) and that $f, \varphi, h, g_{i} \in C^{2}$ for $i=1, \ldots, r$. Let $(\bar{x}, \bar{u}) \in \Xi$ be a trajectory control pair of (4) and $\xi=(y, u) \in \Xi$ be a trajectory control pair of (10) 
such that $V^{2}(\bar{u}, u)$ contains an essentially bounded function. If there is no $w(1) \in R^{L(2)}(1)$ as in Theorem 2, then the conclusion of Theorem 1 is valid with $\alpha_{0}=0$.

For the sake of completeness we also provide a result not involving assumption (12).

Theorem 3: Assume (H1) and that $\varphi, f, h, g_{i} \in C^{2}$ for $i=1, \ldots, r$. Let $(\bar{x}, \bar{u})$ be a weak local minimizer. Then, for any $\xi=(y, u) \in \mathcal{C}(\bar{x}, \bar{u})$, there exists $(\alpha, \beta, p) \in \Lambda(\bar{x}, \bar{u})$ such that for any trajectory-control pair $(w, v) \in \Xi$ of (14) satisfying (17) for every $j \in\{1, \ldots, k\}$ we have

$$
\frac{1}{2} \Omega(\xi, \alpha, \beta, p)+\int_{0}^{1} \mathcal{H}_{u}[t] v(t) \mathrm{d} t \geq 0 .
$$

Example 1: Our first example concerns the classical case of a convex polytope $U$ in $\mathbb{R}^{m}$, that is the convex hull of a finite number of points of $\mathbb{R}^{m}$.

Let $(\bar{x}, \bar{u})$ be a weak local minimizer and suppose that all the assumptions of Theorem 1 hold true. Consider $\xi=$ $(y, u) \in \mathcal{C}(\bar{x}, \bar{u})$. It is not difficult to realize that $0 \in$ $T_{U}^{b(2)}(\bar{u}(t), u(t))$ and so Theorem 1 can be applied. Let $(\alpha, \beta, p) \in \Lambda(\bar{x}, \bar{u})$ be as in Theorem 1. Then, from (9) and the convexity of $U$ we deduce that $-\mathcal{H}_{u}[t]$ is in the normal cone (of convex analysis) to $U$ at $\bar{u}(t)$ for a.e. $t \in[0,1]$. By Lemma 1 we have $\mathcal{H}_{u}[t] u(t)=0$ a.e. Since

$$
U-\bar{u}(t) \subset C_{U}(\bar{u}(t))
$$

we know that $\mathcal{H}_{u}[t] \kappa \geq 0$ for any $\kappa \in U-\bar{u}(t)$. This and the definition of the second-order tangent imply that for almost all $t$ we have $\mathcal{H}_{u}[t] v \geq 0$ for every $v \in T_{U}^{b(2)}(\bar{u}(t), u(t))$. Hence $\Upsilon(u(t), p(t))=0$. Therefore in this case we obtain the classical second-order condition not involving $\Upsilon$ :

$$
y(1)^{*} l_{x x}(\bar{x}(1), \alpha, \beta) y(1)+\int_{0}^{1} \xi(t)^{*} \mathcal{H}^{\prime \prime}[t] \xi(t) \mathrm{d} t \geq 0 .
$$

Example 2: Let $U=([-1,1] \times\{0\}) \cup(\{0\} \times[-1,1]) \subset$ $\mathbb{R}^{2}$. This set can not be expressed as intersection of sets described via inequalities and equalities verifying the usual constraints qualifications at zero. Furthermore, $C_{U}(0)=\{0\}$. Still its first and second-order adjacent tangents can be easily computed. Moreover it is not difficult to realize that for any weak local minimizer $(\bar{x}, \bar{u})$ the second-order condition of Theorem 1 is again (18).

$$
\begin{aligned}
& \text { Example 3: Let } \\
& \qquad \begin{aligned}
U= & \left\{\left(u_{1}, u_{2}\right) \in \mathbb{R}^{2}:\left(u_{1}+1\right)^{2}+u_{2}^{2}=1\right\} \cup \\
& \left\{\left(u_{1}, u_{2}\right) \in \mathbb{R}^{2}:\left(u_{1}-1\right)^{2}+u_{2}^{2}=1\right\} \subset \mathbb{R}^{2} .
\end{aligned}
\end{aligned}
$$

This set can not be expressed as intersection of sets described via inequalities and equalities verifying the usual constraint qualifications at zero. Consider a trajectory-control pair $(\bar{x}, \bar{u}) \in \Xi$ of (4). If $\bar{u}(t) \neq 0$ for some $t \in[0,1]$, then, by [2, Propositions 4.3.7 and 4.7.5],

$T_{U}^{b}(\bar{u}(t))=\left\{u \in \mathbb{R}^{2}:\left\langle\left(\bar{u}_{1}(t)+\operatorname{sgn}\left(\bar{u}_{1}(t)\right), \bar{u}_{2}(t)\right), u\right\rangle=0\right\}$ and for any $u \in T_{U}^{b}(\bar{u}(t))$ we have $T_{U}^{b(2)}(\bar{u}(t), u)=$

$$
\left\{v \in \mathbb{R}^{2}:\left\langle\left(\bar{u}_{1}(t)+\operatorname{sgn}\left(\bar{u}_{1}(t)\right), \bar{u}_{2}(t)\right), v\right\rangle+\frac{1}{2}|u|^{2}=0\right\},
$$

where sgn stands for the sign. On the other hand, it is easy to check that $T_{U}^{b}((0,0))=\{0\} \times \mathbb{R}$ and from [2, Proposition 4.7.5] it follows that for any $\mu \in \mathbb{R}$ we have

$$
T_{U}^{b(2)}((0,0),(0, \mu))=\left\{ \pm \frac{1}{2} \mu^{2}\right\} \times \mathbb{R} .
$$

Under all the assumptions of Theorem 1, for any $\xi=$ $(y, u) \in \mathcal{C}(\bar{x}, \bar{u})$ the second-order condition (13) is satisfied for some $(\alpha, \beta, p) \in \Lambda(\bar{x}, \bar{u})$. Also $T_{U}^{b}(\bar{u}(t))=$ $C_{U}(\bar{u}(t))$ for all $t$ and therefore for a.e. $t \in[0,1]$ such that $\bar{u}(t)=0$, we have $\mathcal{H}_{2}[t]=0$, where $\mathcal{H}_{2}[t]$ denotes the derivative of $\mathcal{H}\left(\bar{x}(t), \bar{u}_{1}(t), \cdot, p(t)\right)$ at $\bar{u}_{2}(t)$ and $\Gamma(\bar{u}(t), p(t))=-\frac{1}{2}\left|\mathcal{H}_{1}[t]\right| u_{2}^{2}(t)$, where $\mathcal{H}_{1}[t]$ denotes the derivative of $\mathcal{H}\left(\bar{x}(t), \cdot, \bar{u}_{2}(t), p(t)\right)$ at $\bar{u}_{1}(t)$. That is the term $\Gamma(\bar{u}(t), p(t))$ may be negative when $\bar{u}(t)=0$.

Proofs of the results announced above are provided in Section V, but first we discuss an implicit function theorem on the metric space of controls.

\section{AN IMPLICIT FUNCTION THEOREM}

Consider the set $\mathcal{U}$ of all measurable $u:[0,1] \rightarrow U$. For any $u_{1}, u_{2} \in \mathcal{U}$ define the distance $d\left(u_{1}, u_{2}\right):=\left\|u_{1}-u_{2}\right\|_{1}$. Then $(\mathcal{U}, d)$ is a complete metric space. It is not difficult to realise that under assumptions $(\mathrm{H} 1),(\mathrm{H} 2)$ to every control $u \in \mathcal{U}$ corresponds a unique solution $x_{u}(\cdot)$ of control system (4) defined on $[0,1]$ and the mapping $\mathcal{U} \ni u \mapsto x_{u} \in$ $W^{1,1}\left([0,1], \mathbb{R}^{n}\right)$ is continuous.

Theorem 4: Assume (H1), (H2), $h \in C^{1}$ and let $(\bar{x}, \bar{u})$ be a trajectory-control pair of (4) with $h(\bar{x}(1))=0$. If (12) holds true, then there exist $\varepsilon>0, C>0$ such that for every trajectory-control pair $(x, u) \in \Xi$ with $\|u-\bar{u}\|_{1}<\varepsilon$ we can find a trajectory-control pair $(\tilde{x}, \tilde{u}) \in \Xi$ of (4) satisfying

$$
h(\tilde{x}(1))=0, \quad\|\tilde{u}-u\|_{1} \leq C|h(x(1))| .
$$

The above result is an implicit function theorem. But, because controls belong to the metric space $\mathcal{U}$, it is not possible to differentiate the end-point map $\mathcal{U} \ni u \rightarrow x_{u}(1)$ and to use the classical inverse mapping theorem as this is usually done in the geometric optimal control. Instead we replace derivatives by variations in the following way:

Let $G: \mathcal{U} \rightarrow \mathbb{R}^{k}$ be defined by $G(u):=h\left(x_{u}(1)\right)$. The first-order contingent variation of $G$ at $u \in \mathcal{U}$ is defined by

$$
G^{(1)}(u):=\operatorname{Limsup}_{\delta \rightarrow 0+} \frac{G\left(B_{\delta}(u)\right)-G(u)}{\delta},
$$

where $B_{\delta}(u)$ denotes the closed ball in $\mathcal{U}$ centered at $u$ of radius $\delta>0$. Though the whole set of variations may be difficult to compute, for our purposes we need only its subset that can be expressed via the reachable set $R^{L}(1)$. The following lemma can be proved by using the needle variations technics.

Lemma 2: Under all the assumptions of Theorem 4 there exist $\varepsilon>0, \rho>0$ such that for every $u \in \mathcal{U}$ satisfying $\|u-\bar{u}\|_{1}<\varepsilon$ we have $\rho B \subset \overline{c o} G^{(1)}(u)$, where $B$ denotes the closed unit ball in $\mathbb{R}^{n}$.

Proof of Theorem 4. By Lemma 2, there exist $\varepsilon>$ $0, \rho>0$ such that for every $u \in \mathcal{U}$ with $d(u, \bar{u})<\varepsilon$ we 
have $\rho B \subset \overline{c o} G^{(1)}(u)$. The inverse mapping [4, Theorem 3.2] on the metric space $\mathcal{U}$ implies the result.

Theorem 4 and [10, Proposition 4.2] together imply

Theorem 5: Assume (H1), that $h, f \in C^{2}$ and let $(\bar{x}, \bar{u}) \in$ $\Xi$ be a trajectory-control pair of (4) with $h(\bar{x}(1))=0$ satisfying (12). Consider a trajectory-control pair $(y, u) \in \Xi$ of (10) and a trajectory-control pair $(w, v) \in \Xi$ of (14) such that $h_{j}^{\prime}(\bar{x}(1)) y(1)=0$ and (17) holds true for every $j \in\{1, \ldots, k\}$. Then for every $\delta>0$, there exists a trajectorycontrol pair $\left(x_{\delta}, u_{\delta}\right)$ of (4) such that

$$
h\left(x_{\delta}(1)\right)=0, \quad \lim _{\delta \rightarrow 0+} \frac{\left\|x_{\delta}-\bar{x}-\delta y-\delta^{2} w\right\|_{\infty}}{\delta^{2}}=0
$$

\section{PROOFS OF THE MAIN RESULTS}

Proof of Theorem 1. Note that (12) implies the surjectivity of $h^{\prime}(\bar{x}(1))$. Consequently, $h^{\prime}(\bar{x}(1))^{*}$ is injective and

$$
\left(\operatorname{ker} h^{\prime}(\bar{x}(1))\right)^{\perp}=\operatorname{Im} h^{\prime}(\bar{x}(1))^{*} .
$$

Fix $\xi=(y, u) \in \mathcal{C}(\bar{x}, \bar{u})$ with $V^{2}(\bar{u}, u) \cap L^{\infty}\left([0,1], \mathbb{R}^{m}\right) \neq$ $\emptyset$. From (5) we obtain

$$
\mathcal{R}(1)+R^{L(2)}(1)=R^{L(2)}(1) .
$$

For every $i \in I_{g}$ consider the open, possibly empty, affine half-space in $\mathbb{R}^{n}$ :

$$
\begin{aligned}
& Q_{i}=\left\{\eta \in \mathbb{R}^{n}: g_{i}^{\prime}(\bar{x}(1)) \eta+\frac{1}{2} y(1)^{*} g_{i}^{\prime \prime}(\bar{x}(1)) y(1)<0\right\}, \\
& Q_{0}=\left\{\eta \in \mathbb{R}^{n}: \varphi^{\prime}(\bar{x}(1)) \eta+\frac{1}{2} y(1)^{*} \varphi^{\prime \prime}(\bar{x}(1)) y(1)<0\right\}
\end{aligned}
$$

and the affine subspace

$\Theta=\left\{\omega \in \mathbb{R}^{n}: h_{j}^{\prime}(\bar{x}(1)) \omega+\frac{1}{2} y(1)^{*} h_{j}^{\prime \prime}(\bar{x}(1)) y(1)=0 \forall j\right\}$.

If there exists $i \in I_{g}$ such that $Q_{i}=\emptyset$, then $g_{i}^{\prime}(\bar{x}(1))=0$ and $y(1)^{*} g_{i}^{\prime \prime}(\bar{x}(1)) y(1) \geq 0$. Set then $\beta=0, \alpha_{i}=1$ and $\alpha_{s}=0$ whenever $s \neq i$. Then the claim of the theorem is verified with $p(\cdot)=0$. Similarly, if $Q_{0}=\emptyset$, then $\varphi^{\prime}(\bar{x}(1))=$ 0 and $y(1)^{*} \varphi^{\prime \prime}(\bar{x}(1)) y(1) \geq 0$. Then set $\beta=0, \alpha_{0}=1$ and $\alpha_{s}=0$ whenever $s \neq 0$ and the claim of the theorem follows again with $p(\cdot)=0$. It remains to consider the case $Q_{i} \neq \emptyset$ for every $i \in I_{g} \cup\{0\}$. Observe that if $Q_{i} \neq \emptyset$ and $g_{i}^{\prime}(\bar{x}(1))=0$, then $Q_{i}=\mathbb{R}^{n}$. Define

$$
I:=\left\{i \in I_{g}: g_{i}^{\prime}(\bar{x}(1)) \neq 0,\left\langle g_{i}^{\prime}(\bar{x}(1)), y(1)\right\rangle=0\right\} .
$$

In the expressions below terms involving $I$ are skipped whenever $I$ is empty. Using the contradiction argument, it is not difficult to deduce from Theorem 5 and the fact that $Q_{i}$ are open that for every $w(1) \in\left(\cap_{i \in I} Q_{i}\right) \cap \Theta \cap R^{L(2)}(1)$, the following variational inequality holds true:

$$
\varphi^{\prime}(\bar{x}(1)) w(1)+\frac{1}{2} y(1)^{*} \varphi^{\prime \prime}(\bar{x}(1)) y(1) \geq 0 .
$$

Let $I=\left\{i_{1}, \ldots, i_{\gamma}\right\}$, where $\gamma \leq r$. Hence zero does not belong to the convex set

$$
\begin{gathered}
\left\{\left(q_{0}-\kappa, q_{i_{1}}-\kappa, \ldots, q_{i_{\gamma}}-\kappa, \theta-\kappa\right): \kappa \in R^{L(2)}(1),\right. \\
\left.\theta \in \Theta, q_{0} \in Q_{0}, q_{i_{j}} \in Q_{i_{j}}, j=1, \ldots, \gamma\right\} .
\end{gathered}
$$

By the separation theorem (and since the above set is a subset of a finite dimensional space), there exist $\zeta^{*}, p_{1}, \zeta_{i}^{*} \in\left(\mathbb{R}^{n}\right)^{*}$ for every $i \in I \cup\{0\}$ not vanishing simultaneously such that

$$
\zeta_{0}^{*}+\Sigma_{i \in I} \zeta_{i}^{*}+\zeta^{*}+p_{1}=0
$$

and

$$
\left\{\begin{array}{l}
\inf \zeta_{0}^{*}\left(Q_{0}\right)+\Sigma_{i \in I} \inf \zeta_{i}^{*}\left(Q_{i}\right)+ \\
\inf \zeta^{*}(\Theta)+\inf p_{1}\left(R^{L(2)}(1)\right) \geq 0
\end{array}\right.
$$

From (22) we get

$$
\inf \left\{\left\langle\zeta^{*}, \kappa\right\rangle: \kappa \in \operatorname{ker} h^{\prime}(\bar{x}(1))\right\}>-\infty .
$$

Hence, by (19), $\zeta^{*}=h^{\prime}(\bar{x}(1))^{*} q$ for some $q \in\left(\mathbb{R}^{k}\right)^{*}$. Set $\beta=-q$. In the same way, by (22) for every $i \in I$ we have

$$
\left.\zeta_{i}^{*} \in\left(\operatorname{ker} g_{i}^{\prime}(\bar{x}(1))\right)^{\perp}=\operatorname{Im} g_{i}^{\prime}(\bar{x}(1))\right)^{*} .
$$

Hence $\zeta_{i}^{*}=\lambda_{i} g_{i}^{\prime}(\bar{x}(1))^{*}$ for some $\lambda_{i} \in \mathbb{R}$. Because for all large $\lambda>0$ we have $-\lambda g_{i}^{\prime}(\bar{x}(1)) \in Q_{i}$, it follows that $\lambda_{i} \leq 0$. Similarly, if $\varphi^{\prime}(\bar{x}(1)) \neq 0$, then $\zeta_{0}^{*}=\lambda_{0} \varphi^{\prime}(\bar{x}(1))^{*}$ for some $\lambda_{0} \leq 0$. Observe next that for every $i \in I$, we have

$$
\sup _{\kappa \in Q_{i}}\left\langle g_{i}^{\prime}(\bar{x}(1)), \kappa\right\rangle=-\frac{1}{2} y(1)^{*} g_{i}^{\prime \prime}(\bar{x}(1)) y(1) .
$$

Therefore

$$
\inf _{\kappa \in Q_{i}}\left\langle\zeta_{i}^{*}, \kappa\right\rangle=\frac{\left|\lambda_{i}\right|}{2} y(1)^{*} g_{i}^{\prime \prime}(\bar{x}(1)) y(1) .
$$

Define $\alpha_{i}=\left|\lambda_{i}\right|$ for all $i \in I$ and $\alpha_{i}=0$ whenever $i \in$ $\{1, \ldots, r\} \backslash I$. Similarly, if $\varphi^{\prime}(\bar{x}(1)) \neq 0$, then

$$
\inf \zeta_{0}^{*}\left(Q_{0}\right)=\frac{\left|\lambda_{0}\right|}{2} y(1)^{*} \varphi^{\prime \prime}(\bar{x}(1)) y(1)
$$

and define $\alpha_{0}=\left|\lambda_{0}\right|$. Observe that if $\varphi^{\prime}(\bar{x}(1))=0$, then $\zeta_{0}^{*}=0$ and set $\alpha_{0}=0$. By (21),

$$
p_{1}=\alpha_{0} \varphi^{\prime}(\bar{x}(1))^{*}+\Sigma_{i=1}^{r} \alpha_{i} g_{i}^{\prime}(\bar{x}(1))^{*}+h^{\prime}(\bar{x}(1))^{*} \beta .
$$

Observe that since $\zeta^{*},\left\{\zeta_{i}^{*}\right\}_{i \in I \cup\{0\}}$ do not vanish simultaneously, $(\alpha, \beta) \neq 0$.

Consider the solution $p(\cdot)$ of the adjoint equation (7)-(8) with $\alpha, \beta$ as defined above.

From (22) it follows that inf $\kappa_{\kappa \in R^{L(2)}(1)}\langle p(1), \kappa\rangle$ is bounded from the below. Since $\mathcal{R}(1)$ is a cone, we deduce from (20) and (22) that $\inf _{\kappa \in \mathcal{R}(1)}\langle p(1), \kappa\rangle \geq 0$. Let $(z, \pi) \in \Xi$ be a trajectory-control pair of (15). Then

$$
\begin{aligned}
\langle p(1), z(1)\rangle & =\int_{0}^{1}(\langle\dot{p}(t), z(t)\rangle+\langle p(t), \dot{z}(t)\rangle) \mathrm{d} t \\
& \left.=\int_{0}^{1}\left\langle p(t), f_{u}[t] \pi(t)\right\rangle\right) \mathrm{d} t \geq 0 .
\end{aligned}
$$

The above inequality and the measurable selection theorem imply that $(\alpha, \beta, p) \in \Lambda(\bar{x}, \bar{u})$.

Consider a trajectory-control pair $(w, v) \in \Xi$ of (14). From the above calculations we obtain

$$
\begin{aligned}
& \frac{\alpha_{0}}{2} y(1)^{*} \varphi^{\prime \prime}(\bar{x}(1)) y(1)+\frac{1}{2} \sum_{j=1}^{k} \beta_{j} y(1)^{*} h_{j}^{\prime \prime}(\bar{x}(1)) y(1)+ \\
& \sum_{i \in I} \frac{\alpha_{i}}{2} y(1)^{*} g_{i}^{\prime \prime}(\bar{x}(1)) y(1)+\langle p(1), w(1)\rangle \geq 0 .
\end{aligned}
$$

On the other hand,

$$
\begin{aligned}
& \langle p(1), w(1)\rangle=\int_{0}^{1}(\langle\dot{p}(t), w(t)\rangle+\langle p(t), \dot{w}(t)\rangle) \mathrm{d} t= \\
& \int_{0}^{1}\left(\left\langle p(t), f_{u}[t] v(t)\right\rangle+\frac{1}{2}\left\langle p(t), \xi(t)^{*} f^{\prime \prime}[t] \xi(t)\right\rangle\right) \mathrm{d} t \\
& =\int_{0}^{1}\left(\mathcal{H}_{u}[t] v(t)+\frac{1}{2}\left\langle\mathcal{H}^{\prime \prime}[t] \xi(t), \xi(t)\right\rangle\right) \mathrm{d} t
\end{aligned}
$$


This yields

$$
\frac{1}{2} \Omega(\xi, \alpha, \beta, p)+\int_{0}^{1} \mathcal{H}_{u}[t] v(t) \mathrm{d} t \geq 0
$$

We next observe that for every $\tilde{v} \in V^{2}(\bar{u}, u)$ there exist

$$
v_{i} \in\left\{v \in L^{\infty}\left([0,1], \mathbb{R}^{m}\right) \mid v(t) \in T_{U}^{b(2)}(\bar{u}(t), u(t)) \text { a.e. }\right\}
$$

such that $\mathcal{H}_{u}[\cdot] v_{i}(\cdot)$ converge to $\mathcal{H}_{u}[\cdot] \tilde{v}(\cdot)$ in $L^{1}([0,1], \mathbb{R})$. Thus

$$
\frac{1}{2} \Omega(\xi, \alpha, \beta, p)+\inf _{v \in V^{2}(\bar{u}, u)} \int_{0}^{1} \mathcal{H}_{u}[t] v(t) \mathrm{d} t \geq 0 .
$$

To get the conclusion (13), the proof becomes very technical, but similar to [7, end of Section 5] and relying on measurable selection theorems.

Proof of Theorem 2. From (16) and Remark 1 (d) we deduce (12). By the proof of Theorem 1 , if $Q_{0}=\emptyset$, then the conclusion of Theorem 1 holds with $\alpha_{0}=1, \beta=0$ and $\alpha_{i}=0$ for all $i \neq 0$. Assume next that $Q_{0} \neq \emptyset$. Also, by our assumption, $Q_{i} \neq \emptyset$ whenever $i \in I_{g}$. From the proof of Theorem 1 we have only to show that $\zeta_{0}^{*} \neq 0$. Assume, by a contradiction, that this is not true and let $w(1)$ be as in the assumptions of our theorem. Then from (21) and (22)

$$
\Sigma_{i \in I} \inf \zeta_{i}^{*}\left(Q_{i}-w(1)\right)+\inf \zeta^{*}(\Theta-w(1)) \geq 0 .
$$

This and the choice of $w(1)$ yield $\zeta_{i}^{*}=0$ for every $i \in I$. Consequently, $p_{1}=-h^{\prime}(\bar{x}(1))^{*} q$ for some $0 \neq q \in\left(\mathbb{R}^{k}\right)^{*}$. This and (22) imply that

$$
\inf h^{\prime}(\bar{x}(1))^{*} q\left(R^{L(2)}(1)\right)=\inf _{\kappa \in R^{L(2)}}\left\langle q, h^{\prime}(\bar{x}(1)) \kappa\right\rangle>-\infty
$$

in contradiction with (16), (20).

Proof of Proposition 1. The assumption of this proposition is equivalent to $\left(\cap_{i \in I_{g}} Q_{i}\right) \cap \Theta \cap R^{L(2)}(1)=\emptyset$. The same arguments as in the proof of Theorem 1 can be applied.

Proof of Theorem 3. By Theorem 1 it is enough to consider the case when 0 is the boundary point of $h^{\prime}(\bar{x}(1))\left(R^{L}(1)\right)$. Then, by the separation theorem, there exists $q \in \mathbb{R}^{k}$ different from zero such that

$$
\min _{\kappa \in R^{L}(1)}\left\langle q, h^{\prime}(\bar{x}(1)) \kappa\right\rangle \geq 0 .
$$

Set $\alpha=0, \beta=q$ and consider the solution $p$ of the adjoint equation (7)-(8). Then, as before, (23) and the measurable selection theorem imply that for a.e. $t \in[0,1]$ and for all $u \in U, \mathcal{H}(\bar{x}(t), u, p(t)) \geq \mathcal{H}(\bar{x}(t), \bar{u}(t), p(t))$. This yields (9). Furthermore, if a trajectory-control pair $(w, v)$ of (14) satisfies (17) for every $j \in\{1, \ldots, k\}$, then

$$
\langle p(1), w(1)\rangle+\frac{1}{2} \sum_{j=1}^{k} \beta_{j} y(1)^{*} h_{j}^{\prime \prime}(\bar{x}(1)) y(1)=0 .
$$

On the other hand, as in the proof of Theorem 1,

$$
\langle p(1), w(1)\rangle=\int_{0}^{1}\left(\mathcal{H}_{u}[t] v(t)+\frac{1}{2}\left\langle\mathcal{H}^{\prime \prime}[t] \xi(t), \xi(t)\right\rangle\right) \mathrm{d} t .
$$

Hence

$$
\begin{aligned}
& \frac{1}{2} \sum_{j=1}^{k} \beta_{j} y(1)^{*} h_{j}^{\prime \prime}(\bar{x}(1)) y(1)+\int_{0}^{1} \mathcal{H}_{u}[t] v(t) \mathrm{d} t \\
& \quad+\frac{1}{2} \int_{0}^{1} \xi(t)^{*} \mathcal{H}^{\prime \prime}[t] \xi(t) \mathrm{d} t=0
\end{aligned}
$$

completing the proof.

\section{CONCLUSIONS}

In this article we have considered an optimal control problem with mixed end point constraints and a general set of controls. It is shown that under a constraint qualification involving the equality constraints and the reachable set of the linear system (11), a second-order necessary condition for weak minima of the same nature as in [7] is verified. When such a constraint qualification is violated, then the abnormal maximum principle holds true. An interesting open question concerns the case when $h^{\prime}(\bar{x}(1))$ is surjective, but (12) is not satisfied. Can some more informative conclusions than those of Theorem 3 or Proposition 1 be deduced in such a case ?

\section{REFERENCES}

[1] Aronna M.S., Bonnans J.F., Dmitruk A.V. and Lotito P., Quadratic order conditions for bang-singular extremals, Numer. Algebra Control Optim., 2, 511-546.

[2] Aubin J.-P. and Frankowska H., Set-Valued Analysis, Birkhäuser, Berlin, 1990.

[3] Bonnans J.F. and Osmolovskii N.P., Second-order analysis of optimal control problems with control and initial-final state constraints, J. of Convex Analysis 17, 885-913.

[4] Frankowska H., High order inverse function theorems, Annales de l'I.H.P. Analyse non linaire, S6 (1989), 283-303.

[5] Frankowska H., Hoehener D. and Tonon D., A second-order maximum principle in optimal control under state constraints, Serdica Mathematical Journal, 39 (2013), 233-270.

[6] Frankowska H. and Osmolovskii N.P., Second-order necessary conditions for a strong local minimum in a problem with state and general control constraints, SIAM J. Control Optim., 56 (2018), 2353-2376.

[7] Frankowska H. and Osmolovskii N.P., Second-order necessary conditions for a strong local minimum in a control problem with general control constraints, Applied Mathematics and Optimization, 80 (2019), 135-164.

[8] Frankowska H., Zhang H. and Zhang X., Stochastic optimal control problems with control and initial-final states constraints, SIAM J. Control Optim., 56 (2018), 1823-1855.

[9] Frankowska H., Zhang H. and Zhang X., Necessary optimality conditions for local minimizers of stochastic optimal control problems with state constraints, Trans. Amer. Math. Soc. (in press).

[10] Hoehener D., Variational approach to second-order optimality conditions for control problems with pure state constraints, SIAM J. Control Optim., 50 (2012), 1139-1173.

[11] Levitin E.S., Milyutin A.A. and Osmolovskii N.P., Conditions of high order for a local minimum in problems with constraints, Russian Math. Surveys, 33 (1978), 97-168.

[12] Maurer H. and Osmolovskii N.P., Second-order conditions for optimal control problems with mixed control-state constraints and control appearing linearly, Proceedings of the 52nd IEEE CDC Conference, Firenze, IEEE Publications (2013), 514-519.

[13] Osmolovskii N.P., Necessary second-order conditions for a weak local minimum in a problem with endpoint and control constraints, J. Math. Anal. and Appl., 457, (2018), 1613-1633.

[14] Osmolovskii N.P. and Maurer H., Applications to Regular and Bang-Bang Control: Second ORder Necessary and SufFICIENT OPTIMALITY CONDITIONS IN CALCULUS OF VARIATIONS and Optimal Control, SIAM, Philadelphia, PA, 2012.

[15] Sarychev A., First and second order sufficient optimality conditions for bang-bang controls, SIAM J. on Control and Optimization, 35, (1997), 315-340.

[16] Vinter R.B., Optimal Control, Birkhäuser Boston, Inc., Boston, MA, 2000. 